\title{
BMJ Open Joint effects of self-reported sleep and modifiable physical activity on risk of dyslipidaemia in women aged 45-55 years: a cross-sectional study
}

\author{
Sha Du, ${ }^{1}$ Yuenan Su, ${ }^{1}$ Dongxue Zhang, ${ }^{1}$ Jing Wu, ${ }^{2}$ Huiqiu Zheng, ${ }^{3}$ \\ Xuemei Wang (i) ${ }^{1}$
}

To cite: Du S, Su Y, Zhang D, et al. Joint effects of selfreported sleep and modifiable physical activity on risk of dyslipidaemia in women aged $45-55$ years: a crosssectional study. BMJ Open 2022;12:e049351. doi:10.1136/ bmjopen-2021-049351

- Prepublication history for this paper is available online. To view these files, please visit the journal online (http://dx.doi. org/10.1136/bmjopen-2021049351).

SD and YS contributed equally.

Received 24 January 2021 Accepted 13 December 2021

Check for updates

(C) Author(s) (or their employer(s)) 2022. Re-use permitted under CC BY-NC. No commercial re-use. See rights and permissions. Published by BMJ.

${ }^{1}$ Department of Health Statistics, School of Public Health, Inner Mongolia Medical University, Hohhot, China

${ }^{2}$ National Institute for Chronic and Non-Communicable Disease Control and Prevention, Chinese Center for Disease Control and Prevention, Beijing, China ${ }^{3}$ Department of Child and Adolescent Health and Health Education, School of Public Health, Inner Mongolia Medical University, Hohhot, China

Correspondence to Professor Xuemei Wang; wangxm_zsu@163.com

\section{ABSTRACT}

Objectives Modifiable physical activity (PA) plays an important role in dyslipidaemia risk in middle-aged women with sleep problems, especially perimenopausal women. We aimed to explore the joint effects of sleep and PA on the risk of dyslipidaemia in women aged $45-55$ years, and the extent to which PA moderated the effect of sleep on the risk of dyslipidaemia.

Design A cross-sectional study.

Setting This study was based on the survey of Chronic Disease and Nutrition Monitoring in Adults in Inner Mongolia in 2015.

Participants 721 women aged $45-55$ years were included.

Outcome measurement PA was measured by the Global Physical Activity Questionnaire. Sleep was measured by questionnaire formulated by the Chinese Center for Disease Control and Prevention. Multivariate logistic regression analyses were performed to determine the joint effects of sleep and PA on dyslipidaemia risk. OR and $95 \% \mathrm{Cl}$ were reported.

Results Among all participants, $60.6 \%$ had sleep problems, $29.0 \%$ had low PA and $41.1 \%$ had dyslipidaemia. Women with sleep problems had higher dyslipidaemia risk than women without sleep problems, irrespective of low, moderate or high $\mathrm{PA}$, with $\mathrm{OR}$ $(95 \% \mathrm{Cl})$ of 4.24 (2.40 to 7.49$), 3.14$ (1.80 to 5.49$)$ and 2.04 (1.20 to 3.48 ), respectively. PA could not completely attenuate the negative association between sleep and dyslipidaemia risk. With PA increased from low to high, the OR of dyslipidaemia decreased by 2.20 . Women with sleep problems and low PA had higher risks of high total cholesterol, high triglyceride, low high-density lipoprotein cholesterol and high low-density lipoprotein cholesterol than women without sleep problems and high PA, with $\mathrm{OR}(95 \% \mathrm{Cl})$ of 2.51 (1.18 to 5.35), 2.42 (1.23 to 4.74), 2.88 (1.44 to 5.74) and 2.52 (1.12 to 5.70$)$, respectively.

Conclusions Among women aged $45-55$ years, the joint effects of self-reported sleep and PA on dyslipidaemia risk were more marked for sleep than for PA. Modifiable PA is a widely accessible and effective intervention to reduce the dyslipidaemia risk in women with sleep problems, particularly among perimenopausal women.
Strengths and limitations of this study

- This study was based on the survey of Chronic Disease and Nutrition Monitoring in Adults, covered urban, agricultural and pastoral areas in Inner Mongolia. The participants were recruited consecutively used multistage stratified cluster random sampling, which were generally representative.

- The study explored the joint effects of sleep and physical activity (PA) on the risk of dyslipidaemia in women aged $45-55$ years, and the extent to which PA moderated the effect of sleep on the risk of dyslipidaemia.

- Due to the cross-sectional design of the study, associations between self-reported sleep, PA and the risk of dyslipidaemia can be inferred but not causality.

\section{INTRODUCTION}

Dyslipidaemia is a major risk factor for cardiovascular disease. ${ }^{12}$ Elevated low-density lipoprotein cholesterol (LDL-C) accounted for 4.32 million deaths and 94.9 million disability-adjusted life years worldwide in $2017 .^{3}$ Furthermore, elevated total cholesterol (TC) and triglyceride (TG) can increase the risk of type 2 diabetes mellitus, ${ }^{4}$ and reduced high-density lipoprotein cholesterol (HDL-C) can worsen the outcomes of patients with coronary heart disease. ${ }^{5}$

Dyslipidaemia includes elevated TC, elevated TG, elevated LDL-C and low HDLC. ${ }^{6}$ Risk factors of dyslipidaemia includes age,${ }^{7}$ low oestrogen levels, ${ }^{8}$ sleep disorder, ${ }^{9}$ smoking ${ }^{10}$ and insufficient aerobic exercise. ${ }^{11}$ Among perimenopausal women, aged 45-55 years, ${ }^{12}{ }^{13}$ with decreasing oestrogen levels, the incidence of dyslipidaemia has increased significantly. ${ }^{14} 15$ The prevalence of dyslipidaemia was reported to be $38.7 \%$ among middle-aged and elderly women in China. ${ }^{16}$ Thus, identifying modifiable risk factors for dyslipidaemia is very important for public health. 
Sleep problems tend to become more common in middle-aged and elderly women, and include difficulty in falling asleep, early awakening and awakening during the night. ${ }^{17-20}$ Poor sleep quality was significantly associated with an increased risk of dyslipidaemia. ${ }^{21} 22$ Poor sleep quality was also correlated with high TG and $\mathrm{TC},{ }^{23}$ but negatively correlated with HDL-C. ${ }^{24}$ A potential mechanism for the associations may be that women in the perimenopausal period, had gradually decreasing hormone levels when their sleep problems occurred, leading to dyslipidaemia. ${ }^{25}$

Physical activity (PA) is a modifiable and conventional influencing factor for dyslipidaemia. Several studies demonstrated that low PA had adverse effects on prevalence of dyslipidaemia. ${ }^{16}{ }^{26}$ HDL-C was found to increase after PA intervention. ${ }^{27}$ PA led to high energy consumption, which reduced the level of TG. ${ }^{28}$ For patients with dyslipidaemia, increased PA significantly reduced their risk of death. ${ }^{29}$

There is some evidence that the association between sleep and PA is bidirectional. Moderate-to-vigorous intensity PA was associated with better sleep quality. ${ }^{30} 31$ Poor sleep quality limited $\mathrm{PA},{ }^{32}$ and sleep problems were associated with insufficient PA. ${ }^{33}$ Therefore, the mechanism for the effects of sleep and PA on dyslipidaemia is very complex.

PA is a modifiable lifestyle factor, but sleep problems are important symptoms of neurological diseases that are difficult to regulate. ${ }^{34}$ Casas $e t a \vec{l}^{5}$ reported significant linear trends in TG and HDL-C among leisuretime activity and sleep quality categories in middle-aged women. However, little is known about the associations of PA, including occupational, transition and leisure-time activity, and sleep with risk of dyslipidaemia in women aged $45-55$ years. This study aimed to explore the joint effects of sleep and PA on the risk of dyslipidaemia in women aged 45-55 years, and the extent to which PA moderated the effect of sleep on the risk of dyslipidaemia to provide some effective suggestions for the management of dyslipidaemia in perimenopausal women.

\section{METHODS}

\section{Design and setting of the study}

The data for this cross-sectional study were collected from the survey of Chronic Disease and Nutrition Monitoring in Adults in Inner Mongolia in 2015, conducted by the Chinese Center for Disease Control and Prevention (CDC). The survey was conducted across eight monitoring sites, covered urban, agricultural and pastoral areas in Inner Mongolia. Participants, aged $\geq 18$ years were selected by multistage-stratified cluster random sampling. All participants received on-site investigations that included questionnaires, anthropometric measurements and fasting blood sample collections.

Women aged $45-55$ years were enrolled in the present study $(n=821)$. Subsequently, 100 women were excluded for missing weight, height or waist circumference data $(\mathrm{n}=25)$, missing serum lipid testing data $(\mathrm{n}=8)$, missing alcohol drinking data $(n=1)$, reporting that they did not drink but indicating alcohol intake $(n=32)$ and having mean total PA time exceeding $1440 \mathrm{~min} /$ day $(\mathrm{n}=34)$. Finally, a total of 721 women were included in the study.

\section{Data collection}

The questionnaire used in the study was from Chinese CDC and evaluated by experts, with high reliability and validity. General information including age, ethnicity, educational level, marital status and residence, lifestyle variables including smoking status, alcohol drinking status, sleep and PA, and history of dyslipidaemia were obtained by trained investigators through face-to-face interviews.

Anthropometric data including height, weight and waist circumference were measured by trained investigators. Fasting venous blood samples were collected by trained laboratory staff members. ${ }^{36}$

\section{Measurements}

Dyslipidaemia definition

Dyslipidaemia was defined as having any one of the following: high TC (serum TC $\geq 240 \mathrm{mg} / \mathrm{dL}$ ), high TG (serum TG $\geq 200 \mathrm{mg} / \mathrm{dL}$ ), high LDL-C (serum LDL-C $\geq 160 \mathrm{mg} / \mathrm{dL}$ ), low HDL-C (serum HDL-C $<40 \mathrm{mg}$ / dL) and self-reported dyslipidaemia diagnosis. ${ }^{37}$

\section{Sleep assessment}

Self-reported sleep was measured by the following questions: during the past 30 days, for more than 3 days per week (including 3 days), did you have any one of the following problems: (1) snoring, asphyxiating or suffocating; (2) difficulty in falling asleep (more than $30 \mathrm{~min}$ ); (3) midway wakefulness twice or more; (4) medication for at least 1 day to help sleep; and (5) waking up early and difficulty in falling asleep again. Participants could respond 'yes' or 'no'. We defined sleep problems as at least one answer of 'yes'. These similar questions of sleep assessment have used in previous studies. ${ }^{1738}$

\section{Assessment of PA}

PA was collected using the Global Physical Activity Questionnaire (GPAQ). ${ }^{39}$ Different domain-specific PAs (work, transportation and recreational activities) were classified into moderate and vigorous intensity. For assessment of total PA, we calculated the metabolic equivalent (MET) minutes per week according to the GPAQ analysis guide $^{40}:$ MET-min $/$ week $=($ moderate minutes $\times 4.0$ METs $)$ + (vigorous minutes $\times 8.0$ METs). Total PA was categorised as low (0-1908 MET-min/week), moderate (1909-5129 MET-min/week) and high ( $\geq 5130 \mathrm{MET}-\mathrm{min} /$ week) ${ }^{41}$

\section{Covariates}

Covariates included age, ethnicity (Han/Mongolian/ other minority), educational level (primary school or lower/junior high school/senior high school and above), marital status (single/married/others) and residence (rural/urban). Lifestyle variables included smoking 
Table 1 Characteristics of sleep and physical activity in women

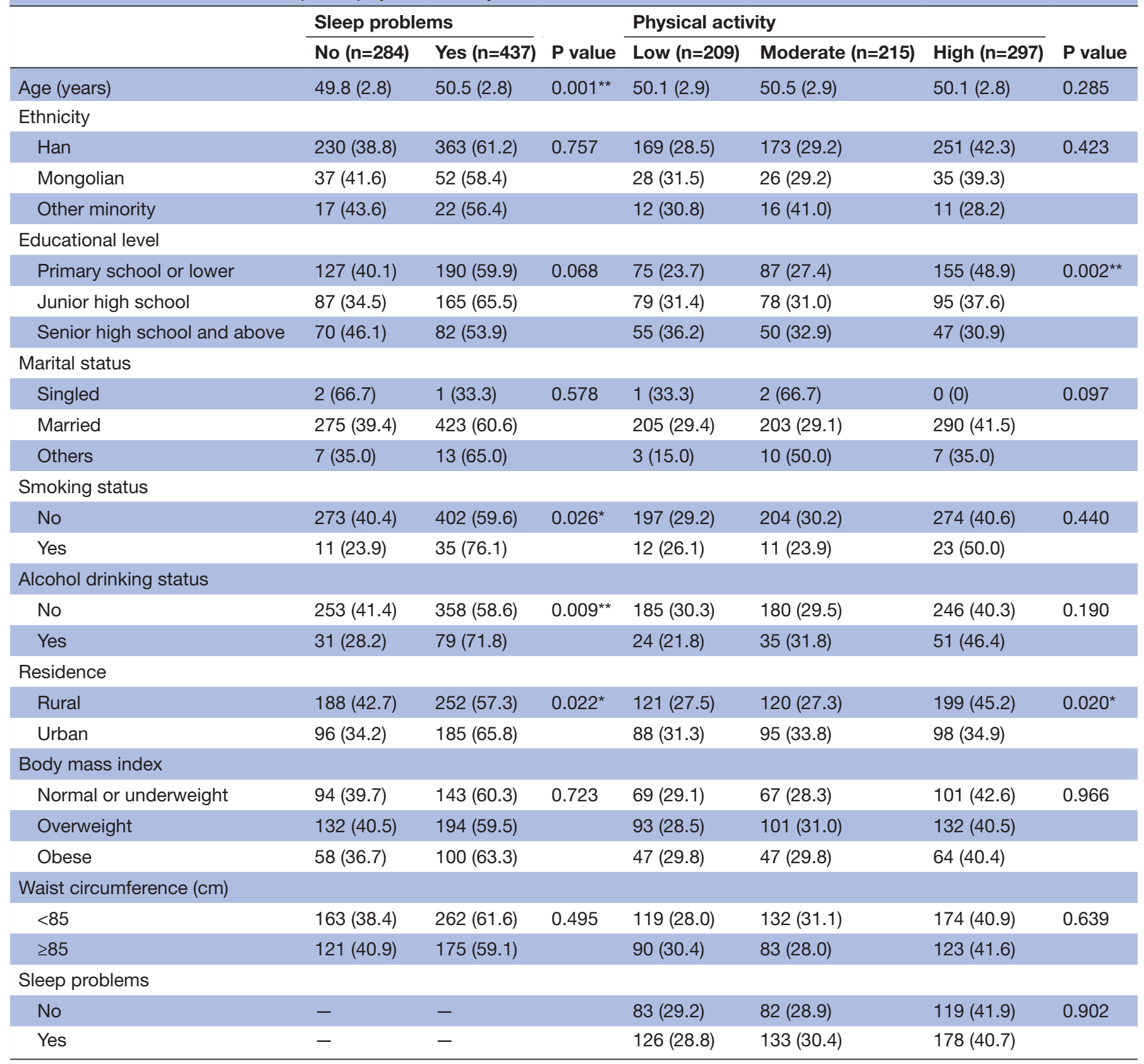

${ }^{*} p<0.05,{ }^{* *} p<0.01$.

status (yes/no) and alcohol drinking status (yes/no). Anthropometric data included height, weight and waist circumference $(<85 \mathrm{~cm}, \geq 85 \mathrm{~cm})$. Body mass index was calculated as weight $(\mathrm{kg}) /$ height $^{2}\left(\mathrm{~m}^{2}\right)$ and categorised as normal or underweight $\left(<24.0 \mathrm{~kg} / \mathrm{m}^{2}\right)$, overweight $\left(24.0-27.9 \mathrm{~kg} / \mathrm{m}^{2}\right)$ or obese $\left(\geq 28.0 \mathrm{~kg} / \mathrm{m}^{2}\right) .{ }^{42}$

\section{Statistical analysis}

Continuous variables were shown as mean \pm SD. Categorical variables were expressed as rate or proportion. The t-test, analysis of variance and $\chi^{2}$ test were used to analyse differences in sleep, PA and dyslipidaemia in the participants with different characteristics. Multivariate logistic regression analyses were conducted to examine the associations of sleep and PA with risk of dyslipidaemia. The results were reported as OR with $95 \%$ CI. We defined $\alpha=0.05$ as the significance level, and two-tailed $\mathrm{p}<0.05$ was considered to indicate statistical significance. All data analyses were performed using IBM SPSS Statistics V.19.0 (IBM Corp).

\section{Patient and public involvement}

This research was done without patient involvement. Patients were not invited to comment on the study design and were not consulted to develop patient-relevant outcomes or interpret the results. Patients were not 
Table 2 Prevalence of dyslipidaemia and its components in women

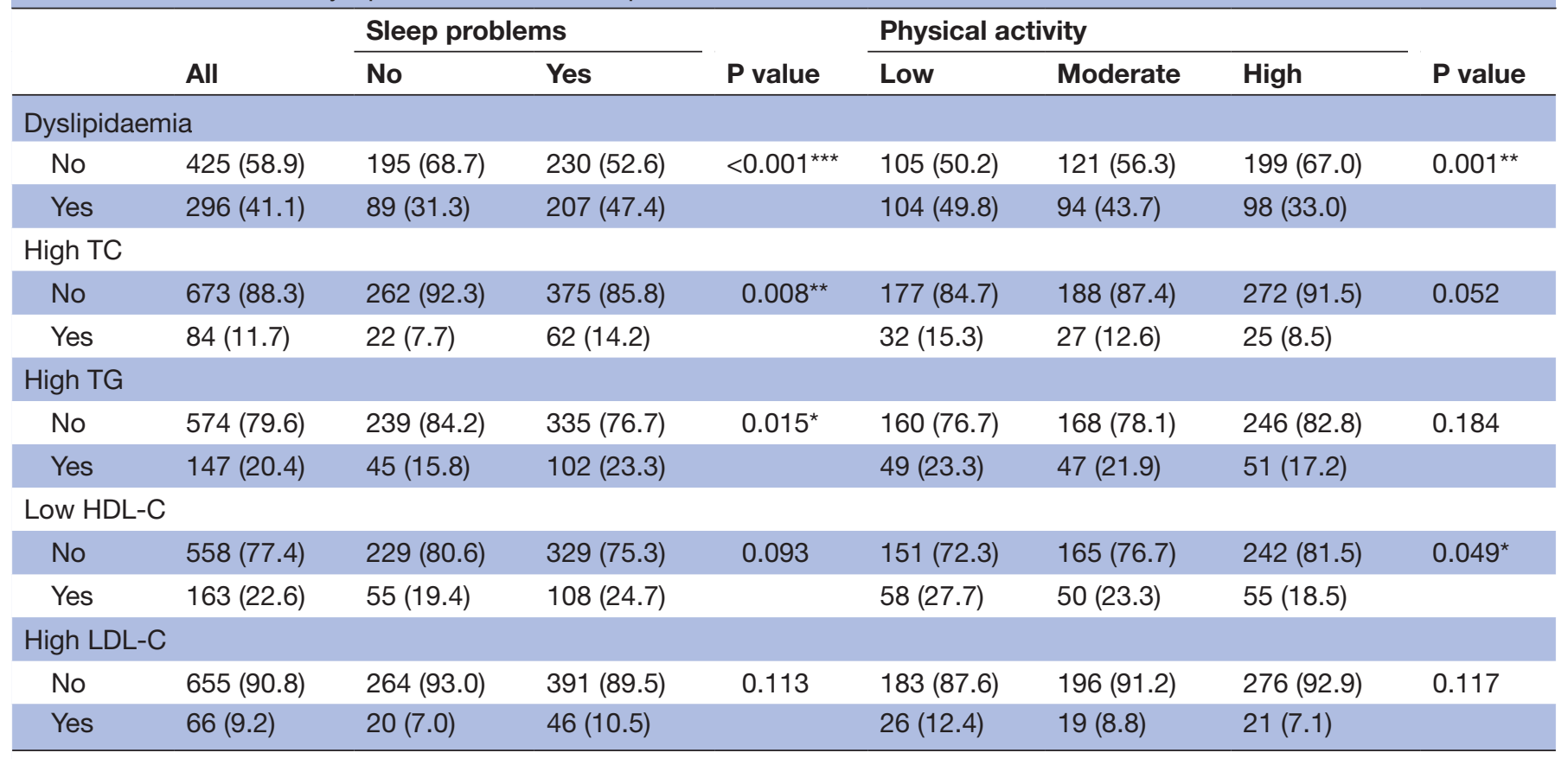

${ }^{*} \mathrm{p}<0.05,{ }^{* *} \mathrm{p}<0.01,{ }^{* * *} \mathrm{p}<0.001$.

HDL-C, high-density lipoprotein cholesterol; LDL-C, low-density lipoprotein cholesterol; TC, total cholesterol; TG, triglyceride.

invited to contribute to the writing or editing of this document for readability or accuracy.

\section{RESULTS}

Demographic characteristics of the participants

Seven hundred and twenty-one women were included in this study and the mean age was 50.2 years $(\mathrm{SD}=2.8$ years). Among all participants, 593 (82.2\%) were Han ethnicity, $430(61.0 \%)$ were from rural areas, $317(44.0 \%)$ had primary school or lower educational level and the majority were married $(96.8 \%)$. Six hundred and eleven $(84.7 \%)$ were non-drinkers, 675 (93.6\%) were non-smokers, 237 $(32.9 \%)$ were normal or underweight and 425 (58.9\%) had waist circumference $<85 \mathrm{~cm}$.

Four hundred and thirty-seven $(60.6 \%)$ had sleep problems and $209(29.0 \%)$ had low PA. There were significant differences in sleep among people with different age, smoking status, alcohol drinking status and residence status, and significant differences in PA among people with different educational level and residence (all $\mathrm{p}<0.05$, table 1$)$. There was no significant difference in PA between the different sleep groups $(\mathrm{p}>0.05$, table 1$)$.

\section{Prevalence of dyslipidaemia and its components}

The prevalence of dyslipidaemia in women with sleep problems was significantly higher than that of women without sleep problems $(47.4 \%$ vs $31.3 \%$; $\mathrm{p}<0.001)$. Women with sleep problems also had higher prevalence of high TC, high TG than women without sleep problems ( $14.2 \%$ vs $7.7 \%, 23.3 \%$ vs $15.8 \%$; all $\mathrm{p} \leq 0.05)$. Women with low PA had a higher prevalence of dyslipidaemia $(49.8 \%$ vs $43.7 \%$ vs $33.0 \%$; $\mathrm{p}=0.001)$, and low HDL-C $(27.7 \%$ vs $23.3 \%$ vs $18.5 \%$; $\mathrm{p}=0.049)$ compared with moderate or high PA. (table 2).

\section{Independent effect of PA and sleep on dyslipidaemia and its} components

In the multivariate logistic analyses, the effects of PA and sleep on risk of dyslipidaemia remained evident. Compared with women without sleep problems, the adjusted OR (95\% CI) for dyslipidaemia, high TC and high TG in women with sleep problems were 2.15 (1.54 to 2.99), 1.81 (1.08 to 3.04) and 1.64 (1.10 to 2.46), respectively. Furthermore, compared with women with high PA, the adjusted OR (95\% CI) for dyslipidaemia in women with low PA was 1.94 (1.32 to 2.84) (table 3).

\section{Joint effects of sleep and PA on the risk of dyslipidaemia and its components}

The participants were categorised into six subgroups according to their joint PA and sleep characteristics. After adjustment for confounders, the joint effects of PA and sleep on dyslipidaemia were significant. Women with sleep problems had higher risk of dyslipidaemia than women without sleep problems, irrespective of low, moderate or high PA, with OR (95\% CI) of 4.24 (2.40 to $7.49), 3.14$ (1.80 to 5.49) and 2.04 (1.20 to 3.48 ), respectively, (figure 1). PA could not completely attenuate the negative association between sleep problems and risk of dyslipidaemia. With PA increased from low to high, the OR of dyslipidaemia decreased by 2.20 (table 4 ).

Likewise, women with sleep problems and low PA had higher risks of high TC, high TG, low HDL-C and high 
Table 3 Independent effect of physical activity and sleep on dyslipidaemia and its components

$\begin{array}{lll}\text { Model } 1 \dagger & \text { Model } 2 \ddagger & \text { P value } \\ \text { OR }(95 \% \mathrm{Cl}) & \text { P value }(95 \% \mathrm{Cl}) & \text { P }\end{array}$

\section{Dyslipidaemia}

Physical activity

\begin{tabular}{|c|c|c|c|c|}
\hline High & 1.00 (reference) & $<0.001^{\star * *}$ & 1.00 (reference) & $0.002^{\star *}$ \\
\hline Moderate & 1.58 (1.10 to 2.27$)$ & & 1.55 (1.06 to 2.27$)$ & \\
\hline Low & 2.01 (1.40 to 2.89 ) & & 1.94 (1.32 to 2.84$)$ & \\
\hline \multicolumn{5}{|c|}{ Sleep problems } \\
\hline No & 1.00 (reference) & $<0.001^{\star \star \star}$ & 1.00 (reference) & $<0.001^{\star \star \star}$ \\
\hline Yes & 1.97 (1.44 to 2.70$)$ & & 2.15 (1.54 to 2.99 ) & \\
\hline \multicolumn{5}{|l|}{ High TC } \\
\hline \multicolumn{5}{|c|}{ Physical activity } \\
\hline High & 1.00 (reference) & $0.017^{*}$ & 1.00 (reference) & 0.059 \\
\hline Moderate & 1.56 (0.88 to 2.78 ) & & 1.48 (0.83 to 2.65$)$ & \\
\hline Low & 1.97 (1.13 to 3.43 ) & & 1.98 (1.13 to 3.47$)$ & \\
\hline \multicolumn{5}{|c|}{ Sleep problems } \\
\hline No & 1.00 (reference) & $0.009^{* *}$ & 1.00 (reference) & $0.025^{*}$ \\
\hline Yes & 1.97 (1.18 to 3.28$)$ & & 1.81 (1.08 to 3.04$)$ & \\
\hline
\end{tabular}

\section{High TG}

Physical activity

\begin{tabular}{|c|c|c|c|c|}
\hline High & 1.00 (reference) & 0.076 & 1.00 (reference) & 0.348 \\
\hline Moderate & 1.35 (0.88 to 2.10$)$ & & 1.30 (0.82 to 2.06$)$ & \\
\hline Low & 1.48 (0.95 to 2.29$)$ & & 1.37 (0.87 to 2.17$)$ & \\
\hline \multicolumn{5}{|c|}{ Sleep problems } \\
\hline No & 1.00 (reference) & $0.015^{*}$ & 1.00 (reference) & $0.015^{\star}$ \\
\hline Yes & $1.62(1.10$ to 2.38$)$ & & 1.64 (1.10 to 2.46$)$ & \\
\hline \multicolumn{5}{|l|}{ Low HDL-C } \\
\hline \multicolumn{5}{|c|}{ Physical activity } \\
\hline High & 1.00 (reference) & $0.014^{*}$ & 1.00 (reference) & 0.083 \\
\hline Moderate & 1.33 (0.87 to 2.05$)$ & & 1.32 (0.84 to 2.07 ) & \\
\hline Low & 1.69 (1.11 to 2.58$)$ & & 1.65 (1.06 to 2.57 ) & \\
\hline \multicolumn{5}{|c|}{ Sleep problems } \\
\hline No & 1.00 (reference) & 0.094 & 1.00 (reference) & 0.073 \\
\hline Yes & 1.37 (0.95 to 1.97$)$ & & 1.43 (0.97 to 2.10$)$ & \\
\hline \multicolumn{5}{|l|}{ High LDL-C } \\
\hline \multicolumn{5}{|c|}{ Physical activity } \\
\hline High & 1.00 (reference) & $0.043^{*}$ & 1.00 (reference) & 0.092 \\
\hline Moderate & 1.27 (0.67 to 2.43 ) & & 1.38 (0.72 to 2.65$)$ & \\
\hline Low & 1.87 (1.02 to 3.42$)$ & & 1.98 (1.07 to 3.65$)$ & \\
\hline \multicolumn{5}{|c|}{ Sleep problems } \\
\hline No & 1.00 (reference) & 0.115 & 1.00 (reference) & 0.064 \\
\hline Yes & 1.55 (0.90 to 2.69$)$ & & 1.69 (0.97 to 2.95$)$ & \\
\hline
\end{tabular}

${ }^{*} p<0.05,{ }^{* *} p<0.01,{ }^{* * *} p<0.001$.

†Model 1: Unadjusted.

$\ddagger$ Model 2: Adjusted for age, ethnicity, education level, marital status, residence, smoking status, alcohol drinking status, waist circumference and physical activity (association of sleep problems) or sleep problems (association of physical activity).

HDL-C, high-density lipoprotein cholesterol; LDL-C, low-density lipoprotein cholesterol; TC, total cholesterol; TG, triglyceride. 


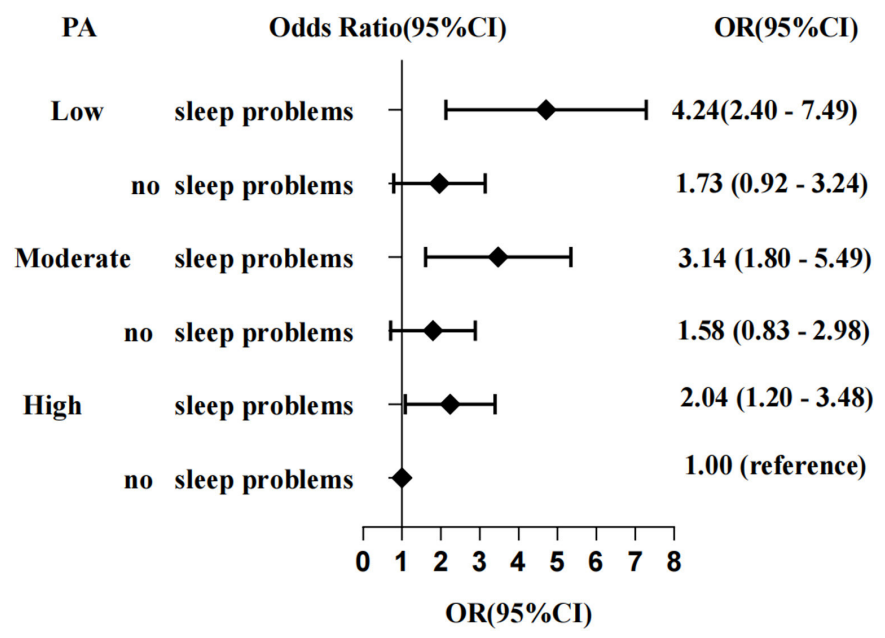

Figure 1 The simple effect of sleep with dyslipidaemia. PA, physical activity.

LDL-C than women without sleep problems and high PA, with OR (95\% CI) of 2.51 (1.18 to 5.35), 2.42 (1.23 to $4.74), 2.88$ (1.44 to 5.74) and 2.52 (1.12 to 5.70), respectively. With PA increased from low to high, the OR of low HDL-C decreased by 0.91 (table 4 ). Sleep problems were associated with increased risks of dyslipidaemia $(\mathrm{OR}=3.14$; 95\% CI: 1.80 to 5.49$)$ and low HDL-C (OR=2.08; 95\% CI: 1.03 to 4.20$)$ among women with moderate PA. In addition, high PA was associated with increased risk of low HDL-C (OR=1.97; 95\% CI: 1.01 to 3.84) among women with sleep problems (figure $2 \mathrm{~A}-\mathrm{D}$ ).

\section{DISCUSSION}

In the present study, the prevalence of dyslipidaemia was $41.1 \%$ in women aged $45-55$ years, $60.6 \%$ of participants had sleep problems and $29.0 \%$ participants had low PA. The prevalence of dyslipidaemia was $47.4 \%$ in women with sleep problems, and significantly higher than that in women without sleep problems.
Low PA and sleep problems were interactively associated with an increased risk of dyslipidaemia in women aged 45-55 years. Furthermore, the effects on dyslipidaemia were more marked for sleep than for PA. We found that PA attenuated but did not eliminate the excess risk of dyslipidaemia associated with sleep problems. With PA increased from low to high, the OR of dyslipidaemia and low HDL-C decreased by 2.20 and 0.91 , respectively.

\section{Comparisons with other studies}

Among middle-aged and older people, PA had significant positive associations with several health conditions, including reduced risks of cardiovascular disease, ${ }^{43}$ diabetes ${ }^{44}$ and hypertension. ${ }^{45}$ Our study showed that high PA was associated with lower risk of dyslipidaemia and its components, included high TC and high TG in women aged 45-55 years. In our study, self-reported sleep was measured by a questionnaire that contained all dimensions of the widely employed Pittsburgh Sleep Quality Index (PSQI), and clearly distinguished whether women had sleep problems. Consistent with our results, previous studies reported that sleep problems measured by the PSQI were associated with the increased risk of dyslipidaemia and its components. ${ }^{2324}$

To date, epidemiological evidence regarding the joint effects of PA and sleep on dyslipidaemia has been limited. Casas $e t a l^{35}$ reported that low leisure-time activity was negatively associated with high TC and low HDL-C. The study pointed out that the joint effects on serum lipids were more marked for leisure-time activity than for sleep quality. Contrary to their results, the effect on risk of dyslipidaemia were more marked for sleep than for PA in the present study. These discrepancies among the findings may be partly explained by differences in the measurement methods for PA and sleep quality. In our study, we measured PA by the GPAQ, which included occupational, transition and leisure-time PA, and used a questionnaire to assess sleep quality. Whereas Casas, et al

Table 4 The $\triangle \mathrm{OR}$ of the dyslipidaemia and its components in women with sleep problems

\begin{tabular}{|c|c|c|c|c|c|}
\hline & \multirow[b]{2}{*}{ Sleep problems } & \multicolumn{3}{|l|}{ Physical activity } & \multirow[b]{2}{*}{$\Delta \mathrm{OR}$} \\
\hline & & Low OR $(95 \% \mathrm{Cl})$ & Moderate OR $(95 \% \mathrm{Cl})$ & High OR (95\% Cl) & \\
\hline \multirow[t]{2}{*}{ Dyslipidaemia } & Yes & $4.24(2.40 \text { to } 7.49)^{*}$ & $3.14(1.80 \text { to } 5.49)^{*}$ & $2.04(1.20 \text { to } 3.48)^{*}$ & 2.20 \\
\hline & No & 1.73 (0.92 to 3.24$)$ & 1.58 (0.83 to 2.98$)$ & 1.00 (reference) & \\
\hline \multirow[t]{2}{*}{ High TC } & Yes & $2.51(1.18 \text { to } 5.35)^{*}$ & 1.65 (0.75 to 3.60$)$ & 0.79 (0.34 to 1.83$)$ & 1.72 \\
\hline & No & 0.65 (0.22 to 1.94$)$ & 0.76 (0.27 to 2.16$)$ & 1.00 (reference) & \\
\hline \multirow[t]{2}{*}{ High TG } & Yes & $2.42(1.23 \text { to } 4.74)^{*}$ & 1.87 (0.94 to 3.71$)$ & 1.56 (0.80 to 3.01$)$ & 0.86 \\
\hline & No & 1.05 (0.47 to 2.37$)$ & 1.49 (0.68 to 3.24$)$ & 1.00 (reference) & \\
\hline \multirow[t]{2}{*}{ Low HDL-C } & Yes & $2.88(1.44 \text { to } 5.74)^{*}$ & $2.08(1.03 \text { to } 4.20)^{*}$ & $1.97(1.01 \text { to } 3.84)^{\star}$ & 0.91 \\
\hline & No & 2.12 (1.00 to 4.49$)$ & 2.00 (0.92 to 4.31$)$ & 1.00 (reference) & \\
\hline \multirow[t]{2}{*}{ High LDL-C } & Yes & $2.52(1.12 \text { to } 5.70)^{*}$ & 1.52 (0.64 to 3.62$)$ & 0.78 (0.32 to 1.92$)$ & 1.74 \\
\hline & No & 0.74 (0.24 to 2.25$)$ & 0.78 (0.25 to 2.38$)$ & 1.00 (reference) & \\
\hline
\end{tabular}

$* \mathrm{p}<0.05$.

HDL-C, high-density lipoprotein cholesterol; LDL-C, low-density lipoprotein cholesterol; TC, total cholesterol; TG, triglyceride. 
(A)

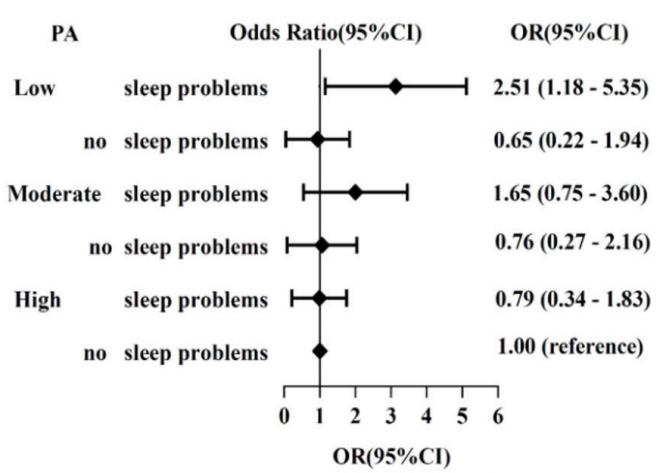

(C)

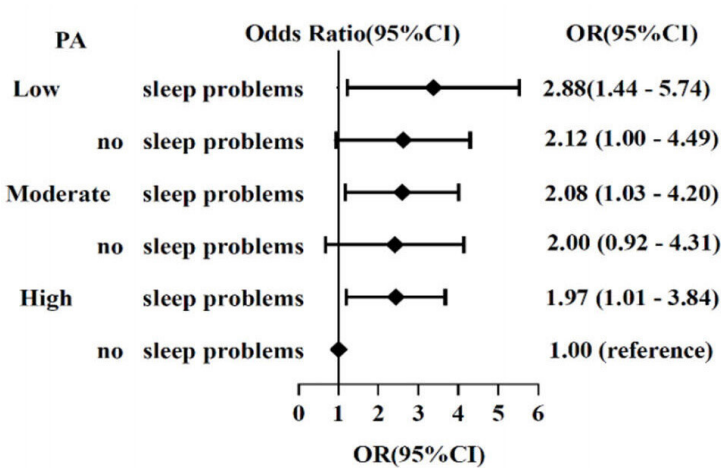

(B)

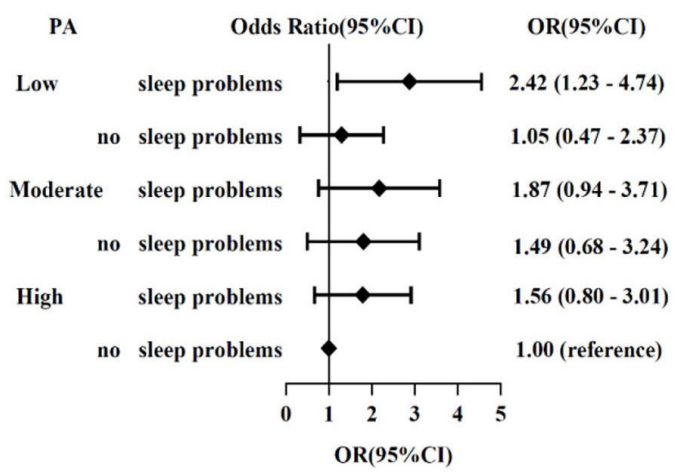

(D)

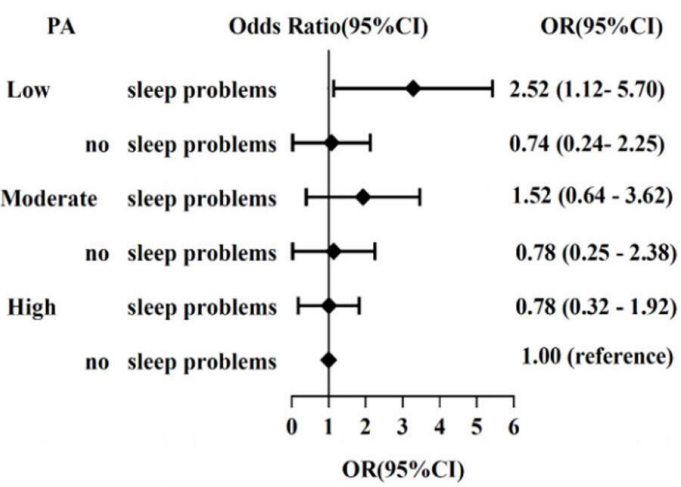

Figure 2 The simple effect of sleep with the components of dyslipidaemia; (A) TC, total cholesterol; (B) TG, triglyceride; (C) HDL-C, high-density lipoprotein cholesterol; (D) LDL-C, low-density lipoprotein cholesterol. PA, physical activity.

only analysed the leisure-time PA, and measured sleep quality by PSQI. What is more, the women participants were aged 45-55 years, who were in the perimenopausal period accompanied by changes in hormone levels. Previous study showed that daily PA and energy expenditure significantly decreased during the perimenopausal period. ${ }^{46}$ Santoro showed that poor sleep quality became more common in perimenopausal women associated with the hormonal changes. ${ }^{47}$ Furthermore, with decreasing oestrogen levels, the risk of dyslipidaemia of perimenopausal women has increased significantly. ${ }^{15}$

There are several potential factors to address for the difference between the simple effects of sleep and PA on the risk of dyslipidaemia. Age was positively associated with risk of dyslipidaemia. ${ }^{9}{ }^{48}$ In our study, women with sleep problems were older, and women with dyslipidaemia were also older. Alcohol drinking has been suggested as a possible risk factor for dyslipidaemia. ${ }^{49}{ }^{50}$ In our study, women with sleep problems were more likely to drink alcohol, which may be another reason why sleep problems had a greater effect on dyslipidaemia.

Our results were consistent with previous studies regarding the interactive associations of sleep and PA with chronic pain, ${ }^{51}$ somatic symptoms,${ }^{52}$ insulin resistance ${ }^{53}$ and depressive symptoms. ${ }^{54}$ All of these studies highlighted the health benefits of PA and suggested that any adverse effects of sleep problems on those outcomes may be reduced by regular PA. In our study, with PA increased from low to high, the OR of dyslipidaemia decreased by
2.20. Meanwhile, contrary to other studies,${ }^{32}{ }^{33}$ there was no significant association between PA and sleep problems, suggesting that increasing PA may not improve sleep quality in middle-aged women.

Modifiable PA has an effective protection on human's health against chronic diseases ${ }^{55-57}$ especially in participants with sleep problems, even if PA did not completely counteract the risk among sleep problems individuals. Modifiable PA are widely accessible and effective intervention to reduce the risk of dyslipidaemia and other chronic diseases in women with sleep problems, especially in perimenopausal women.

\section{Strengths and limitations}

Our study has some strengths and limitations. To our knowledge, there were relatively few studies on the joint effects of sleep and PA on risk of dyslipidaemia. This study was based on a survey of chronic disease and nutrition in Chinese adults, covered urban, agricultural and pastoral areas in Inner Mongolia. The participants were recruited consecutively used multistage cluster random sampling, which were generally representative. However, our study was a cross-sectional study, which only showed the relationship between sleep, PA and the risk of dyslipidaemia, not the causal association. In our study, self-reported sleep was measured by a questionnaire that included five questions. Although, these five questions consist all dimensions of the widely employed PSQI, there were some biases in our results due to no special scale used. 
Therefore, it is necessary to conduct more prospective and experimental research on the joint effects of sleep and PA on the risk of dyslipidaemia in women in the perimenopausal period.

\section{CONCLUSIONS}

In women aged 45-55 years, the joint effects of selfreported sleep and PA on risk of dyslipidaemia were more marked for sleep than for PA. Women aged 45-55 years with sleep problems and low PA should be given more attention. Modifiable PA is a widely accessible and effective intervention to reduce the risk of dyslipidaemia in women with sleep problems, particularly among women during the perimenopausal period. The study can provide some effective suggestions for the management of dyslipidaemia in perimenopausal women. Further research is warranted to identify available services for perimenopausal women to improve their quality of life.

Acknowledgements We thank all participants and investigators for the Chronic Disease and Nutrition Monitoring in Adults in Inner Mongolia in 2015. We also thank Min Yang and Yanling Wang for their contributions to the article, they contributed to revising the manuscript and providing some useful suggestions for review.

Contributors XW contributed to conceiving the study concept and design and was responsible for the overall content as the guarantor. SD and YS analysed the data and wrote the first draft of the paper. All authors (SD, YS, DZ, JW, HZ and XW) contributed to the interpretation of data and were involved revising the manuscript critically for important intellectual content. All authors gave a final approval of the version to be published and agreed to be accountable for all aspects of the work. The authors declared that they had no conflict of interests.

Funding This study was supported by the National Natural Science Foundation of China, grant number 81960603. (Study on the Mechanism of NAMPT Regulated by Hyperglycemia and CpnT Promoting Macrophage Death in Diabetes with Pulmonary Tuberculosis Infection.)

Competing interests None declared.

Patient and public involvement Patients and/or the public were not involved in the design, or conduct, or reporting, or dissemination plans of this research.

\section{Patient consent for publication Not applicable.}

Ethics approval The Chronic Disease and Nutrition Monitoring in Adults in 2015 programme protocols were approved by the Ethical Committee of the National Institute for Chinese Center for Disease Control and Prevention (201519-A). Participants gave informed consent to participate in the study before taking part.

Provenance and peer review Not commissioned; externally peer reviewed.

Data availability statement № data are available.

Open access This is an open access article distributed in accordance with the Creative Commons Attribution Non Commercial (CC BY-NC 4.0) license, which permits others to distribute, remix, adapt, build upon this work non-commercially, and license their derivative works on different terms, provided the original work is properly cited, appropriate credit is given, any changes made indicated, and the use is non-commercial. See: http://creativecommons.org/licenses/by-nc/4.0/.

ORCID iD

Xuemei Wang http://orcid.org/0000-0003-0298-9695

\section{REFERENCES}

1 Baigent C, Keech A, Kearney PM, et al. Efficacy and safety of cholesterol-lowering treatment: prospective meta-analysis of data from 90,056 participants in 14 randomised trials of statins. Lancet 2005;366:1267-78.

2 Nelson $\mathrm{RH}$. Hyperlipidemia as a risk factor for cardiovascular disease. Prim Care 2013;40:195-211.
3 GBD 2017 Risk Factor Collaborators. Global, regional, and national comparative risk assessment of 84 behavioural, environmental and occupational, and metabolic risks or clusters of risks for 195 countries and territories, 1990-2017: a systematic analysis for the global burden of disease study 2017. Lancet 2018;392:1923-94.

4 Zhang M, Zhou J, Liu Y, et al. Risk of type 2 diabetes mellitus associated with plasma lipid levels: the rural Chinese cohort study. Diabetes Res Clin Pract 2018;135:150-7.

5 Zhao Q, Li J, Yang J, et al. Association of total cholesterol and HDL-C levels and outcome in coronary heart disease patients with heart failure. Medicine 2017;96:e6094.

6 National cholesterol education program (NCEP) expert panel on detection, evaluation, and treatment of high blood cholesterol in adults (adult treatment panel III). third report of the National cholesterol education program (NCEP) expert panel on detection, evaluation, and treatment of high blood cholesterol in adults (adult treatment panel III) final report. Circulation 2002;106:3143-421.

7 Ebrahimi $\mathrm{H}$, Emamian $\mathrm{MH}$, Hashemi $\mathrm{H}$, et al. Dyslipidemia and its risk factors among urban middle-aged Iranians: a population-based study. Diabetes Metab Syndr 2016;10:149-56.

8 Stevenson JC, Chines A, Pan K, et al. A pooled analysis of the effects of conjugated estrogens/bazedoxifene on lipid parameters in postmenopausal women from the selective estrogens, menopause, and response to therapy (SMART) trials. J Clin Endocrinol Metab 2015:100:2329-3238.

$9 \mathrm{Wu} \mathrm{H}$, Xiong L, Xu Q, et al. Higher serum triglyceride to highdensity lipoprotein cholesterol ratio was associated with increased cardiovascular mortality in female patients on peritoneal dialysis. Nutr Metab Cardiovasc Dis 2015;25:749-55.

10 Wang Z, Wang D, Wang Y. Cigarette smoking and adipose tissue: the emerging role in progression of atherosclerosis. Mediators Inflamm 2017;2017:3102737.

11 Donley DA, Fournier SB, Reger BL, et al. Aerobic exercise training reduces arterial stiffness in metabolic syndrome. J Appl Physiol 2014;116:1396-404.

12 Nelson HD. Menopause. Lancet 2008;371:760-70.

$13 \mathrm{Li} \mathrm{L,} \mathrm{Wu} \mathrm{J,} \mathrm{Pu} \mathrm{D,} \mathrm{et} \mathrm{al.} \mathrm{Factors} \mathrm{associated} \mathrm{with} \mathrm{the} \mathrm{age} \mathrm{of} \mathrm{natural}$ menopause and menopausal symptoms in Chinese women. Maturitas 2012;73:354-60.

14 Chinese College of Cardiovascular Physician Women Physician Committee, Chinese Society of Cardiology Women's Heart Health Group. [Chinese expert consensus on management of dyslipidemia in postmenopausal women]. Zhonghua Xin Xue Guan Bing Za Zhi 2014:42:279-83.

15 Cortez-Dias N, Robalo Martins S, Belo A, et al. Em nome DOS Investigadores do Estudo VALSIM. Characterization of lipid profile in primary health care users in Portugal. Rev Port Cardiol 2013;32:987-96.

16 Li Y, Zhao L, Yu D, et al. The prevalence and risk factors of dyslipidemia in different diabetic progression stages among middleaged and elderly populations in China. PLoS One 2018;13:e0205709.

17 Sagayadevan V, Abdin E, Binte Shafie S, et al. Prevalence and correlates of sleep problems among elderly Singaporeans. Psychogeriatrics 2017;17:43-51.

18 Bruyneel M. Sleep disturbances in menopausal women: aetiology and practical aspects. Maturitas 2015;81:406-9.

19 Boyle A, Melville CA, Morrison J, et al. A cohort study of the prevalence of sleep problems in adults with intellectual disabilities. J Sleep Res 2010;19:42-53.

20 Harvey MT, Baker DJ, Horner RH, et al. A brief report on the prevalence of sleep problems in individuals with mental retardation living in the community. J Posit Behav Interv 2003:5:195-200.

21 Hung C-M, Li Y-C, Chen H-J, et al. Risk of dementia in patients with primary insomnia: a nationwide population-based case-control study. BMC Psychiatry 2018;18:38.

22 Medic G, Wille M, Hemels ME. Short- and long-term health consequences of sleep disruption. Nat Sci Sleep 2017;9:151-61.

23 Wan Mahmood WA, Draman Yusoff MS, Behan LA, et al. Association between sleep disruption and levels of lipids in caucasians with type 2 diabetes. Int J Endocrinol 2013;2013:341506.

24 Hung $\mathrm{H}-\mathrm{C}$, Yang $\mathrm{Y}-\mathrm{C}, \mathrm{Ou} \mathrm{H}-\mathrm{Y}$, et al. The association between self-reported sleep quality and metabolic syndrome. PLoS One 2013;8:e54304

25 Takahashi TA, Johnson KM. Menopause. Med Clin North Am 2015;99:521-34.

26 Liu X, Yu S, Mao Z, et al. Dyslipidemia prevalence, awareness, treatment, control, and risk factors in Chinese rural population: the Henan rural cohort study. Lipids Health Dis 2018;17:119.

27 Nybo L, Sundstrup E, Jakobsen MD, et al. High-intensity training versus traditional exercise interventions for promoting health. Med Sci Sports Exerc 2010;42:1951-8. 
28 LeMura LM, von Duvillard SP, Andreacci J, et al. Lipid and lipoprotein profiles, cardiovascular fitness, body composition, and diet during and after resistance, aerobic and combination training in young women. Eur J Appl Physiol 2000;82:451-8.

29 Kokkinos PF, Faselis C, Myers J, et al. Interactive effects of fitness and statin treatment on mortality risk in veterans with dyslipidaemia: a cohort study. Lancet 2013;381:394-9.

30 Christie AD, Seery E, Kent JA. Physical activity, sleep quality, and self-reported fatigue across the adult lifespan. Exp Gerontol 2016;77:exger.2016.02.001:7-11.

31 Borges-Cosic M, Aparicio VA, Estévez-López F, et al. Sedentary time, physical activity, and sleep quality in fibromyalgia: the al-Ándalus project. Scand J Med Sci Sports 2019;29:266-74.

32 Štefan L, Vrgoč G, Rupčić T, et al. Sleep duration and sleep quality are associated with physical activity in elderly people living in nursing homes. Int J Environ Res Public Health 2018;15:2512.

33 Štefan L, Sporiš G, Krističević T, et al. Associations between sleep quality and its domains and insufficient physical activity in a large sample of Croatian young adults: a cross-sectional study. BMJ Open 2018;8:e021902.

34 Porter VR, Buxton WG, Avidan AY. Sleep, cognition and dementia. Curr Psychiatry Rep 2015;17:97.

35 Casas RS, Pettee Gabriel KK, Kriska AM, et al. Association of leisure physical activity and sleep with cardiovascular risk factors in postmenopausal women. Menopause 2012;19:413-9.

36 Pang SJ. Associations of lipid parameters and phospholipid profiles with insulin resistance among adults. Chinese Center for Diseases Control and Prevention 2018.

37 Kopin L, Lowenstein C. Dyslipidemia. Ann Intern Med 2017;167:ITC81-96.

38 Olinto MTA, Garcez A, Henn RL, et al. Sleep-related problems and minor psychiatric disorders among Brazilian shift workers. Psychiatry Res 2017;257:412-7.

39 World Health Organization. Global physical activity questionnaire (GPAQ). Available: https://www.who.int/ncds/surveillance/steps/ GPAQ_EN.pdf

40 World Health Organization. Global physical activity questionnaire (GPAQ) analysis guide. Available: https://www.who.int/ncds/ surveillance/steps/resources/GPAQ_Analysis_Guide.pdf

41 Yu ZM, Parker L, Dummer TJB. Depressive symptoms, diet quality, physical activity, and body composition among populations in nova Scotia, Canada: report from the Atlantic partnership for tomorrow's health. Prev Med 2014;61:106-13.

42 Xu W, Zhang H, Paillard-Borg S, et al. Prevalence of overweight and obesity among Chinese adults: role of adiposity indicators and age. Obes Facts 2016;9:17-28.
43 de Souto Barreto P, Cesari M, Andrieu S, et al. Physical activity and incident chronic diseases: a longitudinal observational study in 16 European countries. Am J Prev Med 2017;52:373-8.

44 Marques $\mathrm{A}$, Peralta $\mathrm{M}$, Sarmento $\mathrm{H}$, et al. Associations between vigorous physical activity and chronic diseases in older adults: a study in 13 European countries. Eur J Public Health 2018;28:950-5.

45 Huai $\mathrm{P}$, Xun $\mathrm{H}$, Reilly $\mathrm{KH}$, et al. Physical activity and risk of hypertension: a meta-analysis of prospective cohort studies. Hypertension 2013;62:1021-6.

$46 \mathrm{Kim} \mathrm{J-S,} \mathrm{Choe} \mathrm{J-P,} \mathrm{Park} \mathrm{J-H,} \mathrm{et} \mathrm{al.} \mathrm{The} \mathrm{comparison} \mathrm{of} \mathrm{physical}$ activity, sedentary behavior, and mental health between early menopausal women and age-matched General middle-aged women. Int J Environ Res Public Health 2021;18:7256.

47 Santoro N. Perimenopause: from research to practice. J Women Health 2002;25:332-9.

48 Florez H, Silva E, Fernández V, et al. Prevalence and risk factors associated with the metabolic syndrome and dyslipidemia in white, black, Amerindian and mixed Hispanics in Zulia state, Venezuela. Diabetes Res Clin Pract 2005;69:63-77.

49 Pintó X, Valdivielso P, Perez de Juan JM, et al. Predictive factors of achieving therapeutic goals of hypertriglyceridemia. Curr Med Res Opin 2014;30:19-26.

50 Yang J, Ye J, Guo Q, et al. The joint effects of smoking and alcohol drinking on lipid-related indices among Chinese malescomparing exercise and non-exercise groups. Subst Use Misuse 2018;53:2431-8.

51 Mork PJ, Vik KL, Moe B, et al. Sleep problems, exercise and obesity and risk of chronic musculoskeletal pain: the Norwegian HUNT study. Eur J Public Health 2014;24:924-9.

52 Ablin JN, Clauw DJ, Lyden AK, et al. Effects of sleep restriction and exercise deprivation on somatic symptoms and mood in healthy adults. Clin Exp Rheumatol 2013;31:S53-9.

53 Zuo H, Shi Z, Yuan B, et al. Interaction between physical activity and sleep duration in relation to insulin resistance among non-diabetic Chinese adults. BMC Public Health 2012;12:247.

54 Kaseva K, Dobewall H, Yang X, et al. Physical activity, sleep, and symptoms of depression in Adults-Testing for mediation. Med Sci Sports Exerc 2019;51:1162-8.

55 Dao-Tran T-H, Seib C. Prevalence and correlates of sleep disturbance among older women in Vietnam. J Clin Nurs 2018;27:3307-13.

56 Andersson C, Lyass A, Larson MG, et al. Physical activity measured by accelerometry and its associations with cardiac structure and vascular function in young and middle-aged adults. J Am Heart Assoc 2015;4:e001528.

57 Goto C, Higashi Y, Kimura M, et al. Effect of different intensities of exercise on endothelium-dependent vasodilation in humans: role of endothelium-dependent nitric oxide and oxidative stress. Circulation 2003;108:530-5. 